Article

\title{
Anti-Skin-Aging Activity of a Standardized Extract from Panax ginseng Leaves In Vitro and In Human Volunteer
}

\author{
Seoungwoo Shin, Jung-A Lee, Dahee Son, Deokhoon Park and Eunsun Jung * \\ Biospectrum Life Science Institute, U-TOWER 18th FL, 767, Sinsu Ro, Suji Gu, Yongin City, \\ Gyunggi Do 16827, Korea; biost@biospectrum.com (S.S.); biofk@biospectrum.com (J.-A.L.); \\ biouk@biospectrum.com (D.S.); pdh@biospectrum.com (D.P.) \\ * Correspondence: bioso@biospectrum.com; Tel.: +82-31-698-3122; Fax: +82-31-698-3123
}

Academic Editor: Enzo Berardesca

Received: 11 April 2017; Accepted: 25 May 2017; Published: 1 June 2017

\begin{abstract}
Ginseng leaves contain high saponin composition and content, but are used less often than the root part. To develop a use for the leaves that exploits their properties, we studied ginseng leaves as the raw material of anti-aging cosmetics. This study highlights an assessment of the cellular factivity and clinical efficacy of ginseng leaf extract, providing necessary information relevant to the development of new cosmetic products. Panax ginseng leaf purified extracts (PGLE) were shown to have high contents of $\mathrm{Rb} 3$ and $\mathrm{Rb} 2$. Rb3, the major chemical components of PGLE, promoted collagen synthesis though the activation of transforming growth factor- $\beta$ (TGF- $\beta$ ) in human skin fibroblast cells. In addition, the possibility of PGLE as an anti-skin-aging agent has also been clinically validated. Our analysis of the crow's feet wrinkle showed that there was a decrease in the depth of deep furrows in the region of interest (RI) treated with PGLE lotion over an eight-week period. Based on these results, we suggest the possibility that PGLE, having high levels of Rb3, be considered as an attractive, wrinkle-reducing candidate for topical application.
\end{abstract}

Keywords: Panax ginseng leaf; ginsenoside Rb3; collagen synthesis; anti-wrinkle

\section{Introduction}

Aging of the skin is primarily related to reductions in the levels of Type I collagen, which is the principal component of skin dermis. Type I collagen is the main structural component of the extracellular matrix (ECM), which is known to perform a pivotal function in the maintenance of the structure of the skin dermis [1]. Several molecules have been reported to augment type I collagen synthesis, namely, transforming growth factor- $\beta$ (TGF- $\beta$ ) and fibroblasts growth factor 2 (FGF2). Both the quantity and quality of extracellular collagen are determined by the balance existent between degradation and synthesis. Degradation appears to be mediated by matrix metalloproteinases (MMPs), as well as by endogenous tissue inhibitors (TIMPs). To prevent the skin from aging or wrinkle, natural phytochemical sources are desirable. Plant extracts rich in phytochemicals, like flavonoids, phenolic acids, saponins and alkaloids, having collagen synthesis activity, are being widely used for the development of anti-skin-aging topical cosmetic products.

Panax ginseng C.A Meyer (Asian ginseng) has been used for centuries in many oriental countries as a medicinal plant, and as a functional food that promotes health. Extensive reports have determined that ginseng has many pharmacological effects on the immune, cardiovascular, endocrine, and central nervous systems [2]. Ginsenosides are the main active constituents in Panax ginseng. Panax ginseng root is considered the most important part of the plant for medicinal purpose, and most studies on its ginsenosides have focused on the root. However, several studies have reported that the leaf exhibited 
higher contents of ginsenoside than the main root, and that the content of PPD-type ginsenosides was higher than that of PPT-type ginsenosides [3-5]. The leaf contained high amounts of ginsenosides $\mathrm{Rb} 3$, while the main root contained high amounts of ginsenoside Rb1 and Rc. Moreover, Rb3 was only detected in the leaf [6].

In this study, we prepared standardized extract (Panax ginseng leaf purified extracts (PGLE)) from the dried leaves of Panax ginseng. PGLE was further analyzed by HPLC for the identification of major ginsenoside components (Figure 2 and Table 2). The dominant chemical class in PGLE is PPD (69.42\%), with the main two ginsenoside components $\mathrm{Rb} 3$ and $\mathrm{Rb} 2$ comprising 55.72 and 12.07\%, respectively. These extracts were evaluated for collagen synthesis activity in vitro, and the anti-wrinkle effect of formulated lotion containing PGLE in vivo.

\section{Materials and Methods}

\subsection{Cell Line and Cell Culture}

Human dermal fibroblasts (derived from neonatal foreskin) were purchased from American Type Cell Collection (ATCC). The cells were cultured in Dulbecco's Modified Eagle's Medium containing $10 \%$ fetal bovine serum (FBS) (Gibco-BRL, Sparks, MD, USA) and penicillin streptomycin at $37^{\circ} \mathrm{C}$ in a humidified atmosphere containing 95\% air/5\% carbon dioxide [7].

\subsection{Chemicals and Reagent}

The standards of ginsenosides Rb1, Rb2, Rb3, Rc, Rd, Re, Rf, Rg1, Rg2, Rg3, Rh1 and Rh2 were purchased from Ambo Institute (Daejeon, Korea). HPLC-grade Acetonitrile, ethanol and water were purchased from Merck (Darmstadt, Germany). TGF- $\beta$ was purchased from R\&D Systems (Minneapolis, MN, USA). 3-[4,5-dimethylthiazol-2yl]-2,5-diphenyltetrazolium bromide (MTT) was purchased from Sigma (St. Louis, MO, USA). All other chemicals used were of analytical grade.

\subsection{Preparation of the Extract}

The leaf of Panax ginseng was purchased from Geumsan (Korea). The air-dried powdered leaf (1 kg) of ginseng was extracted with $10 \mathrm{~L} \mathrm{70 \%} \mathrm{aqueous} \mathrm{ethanol} \mathrm{at} \mathrm{room} \mathrm{temperature} \mathrm{and} \mathrm{the} \mathrm{supernatant} \mathrm{was}$ collected by filtration. The ethanol was removed by rotary vacuum evaporator (Heidolph, Schwabach, Germany) and the extract was lyophilized (410 g). The 70\% ethanol extract was refluxed with alkaline water ( $\mathrm{pH} 2.5-3.5)$ for $12 \mathrm{~h}$ at $60^{\circ} \mathrm{C}$. The filtrate was partitioned with n-butanol (1:1, 3 times). The dried n-butanol fraction (55 g) was subjected to Diaion HP-20 column. The column was eluted with 30\% ethanol first, followed by $100 \%$ ethanol, and the eluent was collected and concentrated. The dried HP column fraction ( $38.5 \mathrm{~g}$ ) was subjected to open liquid chromatography (Silica-gel, $50 \times 600 \mathrm{~mm}$ ) and eluted with Ethyl acetate-Ethanol (20:1, 10:2, 1:9) to afford 3 fractions (F1-F3). The silica-gel fraction 2-3 was isolated by preparative reversed-phase HPLC (Luna, $250 \times 30.00 \mathrm{~mm}$; $5 \mu \mathrm{m}$ particle size; $15 \mathrm{~mL} / \mathrm{min}$; UV detection at $200 \mathrm{~nm}$ ) eluted with $\mathrm{ACN} / \mathrm{H}_{2} \mathrm{O}$ gradient system, which was shown by HPLC to contain $70 \%$ total ginsenoside. This novel purified extract is obtained only with food-grade solvent (i.e., water and ethanol — it has been Ecocert validated) and is then purified by chromatography. The final result is a standardized extract containing two main ginsenosides (over 65\%) of the extract in weight. The extract was characterized by a high-performance liquid chromatography (HPLC) method for the determination of ginsenoside Rb3 and Rb2.

\subsection{HPLC Analysis of Ginsenoside}

The amounts of ginsenoside were quantitative analyzed by HPLC. A Shimadzu (Kyoto, Japan) HPLC system with a CBM-20A Controller, SPD-M20A PDA Detector, LC-20AD pump, and SIL-20A Autosampler was used for analysis of the ginsenoside. Data acquisition was achieved using the Shimadzu Lab Solution. All chromatographic separations were conducted on a Luna C18(2) 100A column $(250 \mathrm{~mm} \times 4.6 \mathrm{~mm}, 5 \mu \mathrm{m}$, Phenomenex, Torrance, CA, USA) with column oven temperature 
maintained at $37{ }^{\circ} \mathrm{C}$. The mobile phase consisted of water (A) and acetonitrile (B). Elution was performed at a flow rate of $1 \mathrm{~mL} / \mathrm{min}$ in a binary gradient mode. The solvent gradient changed according to the following schedule: from 80\%(A):20\%(B) to $60 \%(\mathrm{~A}): 40 \%(\mathrm{~B})$ in $35 \mathrm{~min}$; to $10 \%(\mathrm{~A}): 90 \%(\mathrm{~B})$ in $15 \mathrm{~min}$; maintained for $5 \mathrm{~min}$, followed by $10 \mathrm{~min}$ of column re-equilibrium with total time of $75 \mathrm{~min}$. Chromatograms were acquired at $200 \mathrm{~nm}$ by UV detector.

\subsection{Cell Viability Assay}

The cells were cultured in Dulbecco's Modified Eagle's Medium (DMEM) containing 10\% fetal bovine serum (FBS) and penicillin streptomycin at $37^{\circ} \mathrm{C}$ in a humidified atmosphere containing $95 \%$ air $/ 5 \%$ carbon dioxide. Cells were seeded on 24 -well plates. After $24 \mathrm{~h}$, cells were cultured in DMEM under serum-free conditions for $48 \mathrm{~h}$ in the presence of different concentrations of PGLE. The cytotoxicity of PGLE was determined using 3-[4,5-dimethylthiazol-2yl]-2,5-diphenyltetrazolium bromide (MTT) reduction to the corresponding blue formazan by viable cells. The level of blue formazan was measured spectrophotometrically and used as an indirect index of cell density. Briefly, cells were exposed to MTT $(1 \mathrm{mg} / \mathrm{mL})$ for $3 \mathrm{~h}$ at $37^{\circ} \mathrm{C}$. The medium was removed, and the cells were solubilized with dimethyl sulfoxide. After complete solubilization, the presence of blue formazan was evaluated spectrophotometrically by measuring the absorbance at $540 \mathrm{~nm}$ (reference, $620 \mathrm{~nm}$ ) with an enzyme-linked immunosorbent assay (ELISA) plate reader. Viability was expressed as a percentage of the control.

\subsection{Quantitative Detection of Type I Collagen}

The quantity of type I collagen in the cells was determined using a commercially available kit (Takara Bio Inc., Kyoto, Japan). This kit is capable of detecting procollagen type I carboxy-terminal peptide (PIP) using polyclonal antibodies, rather than directly measuring collagen. Human dermal fibroblast cells were then incubated in either the presence or the absence of PGLE for $48 \mathrm{~h}$, and then the culture supernatants were harvested and measured with a sandwich immunoassay kit, which was utilized in accordance with the manufacturer's instructions (Takara Bio Inc., Kyoto, Japan). The measurement was performed with a microplate at $450 \mathrm{~nm}$.

\subsection{Study Design}

This study was designed as a randomized, double-blind study to assess the clinical effects of the use of lotion containing $0.05 \%$ PGLE on periocular wrinkles over an 8-week period. This study was conducted in agreement with the "Declaration of Helsinki" and approved by the Ethics Committee of the Korea Dermatology Research Institute in Seongnam, Korea.

\subsection{Human Volunteers}

Twenty-four healthy Korean female volunteers, aged 35-59 years (mean $\pm \mathrm{SD}=50.04 \pm 6.62$ years) and with fine wrinkles in the skin around the eyelids, were chosen for this study and gave signed, informed consent. All volunteers exposed themselves regularly to sunshine and were not allowed to use cosmetic products during the 8-week treatment. Volunteers were also asked not to use make-up on the day of measurement. Subjects with a known allergy to any ingredient in the lotion or those who had used topical applications of any lotion on the region of interest (RI) during the preceding month were not included. No topical application of any other product on the RI was permitted. The other exclusion criteria were: acute or chronic illness likely to necessitate a treatment with corticoids; topical vitamin A acid or a-hydroxy acid treatment during the month before the beginning of the study; and disorders resulting from excessive consumption of alcohol or toxic substances. All volunteers applied precisely measured amounts $(0.1 \mathrm{~g})$ of lotion-containing $0.05 \%$ PGLE or of the equivalent control lotion containing vehicle. The probands that presented with signs of skin aging on the skin regions were selected for treatment and evaluation. 


\subsection{Preparation of Lotion Containing $0.05 \%$ PGLE}

Oil-in-water lotion with or without $0.05 \%$ PGLE was prepared. The lotion contained several principal ingredients including glycerin, shea butter, 1,2-Haxandiol, ethylhexylglycerin, Cetearyl alcohol, mineral oil and beeswax (Table 1). Lotion with or without PGLE $(0.05 \%)$ was applied to the periorbital region.

Table 1. Formulation of PGLE-containing lotion and placebo lotion.

\begin{tabular}{cccc}
\hline Phase & INCI Name & \%W/W Placebo & \%W/W Test \\
\hline \multirow{2}{*}{ A } & Deionized Water & To 100 & To 100 \\
& Glycerin & 3.00 & 3.00 \\
\hline \multirow{4}{*}{ B } & Shea Butter & 3.00 & 3.00 \\
& Cetearyl Alcohol & 2.00 & 2.00 \\
& Beeswax & 2.00 & 2.00 \\
& Cetearyl Olivate (and) & 2.00 & 2.00 \\
& Sorbitan Olivate & 6.00 & 6.00 \\
\hline C & Mineral Oil & & 0.05 \\
\hline \multirow{2}{*}{ Panax ginseng leaf extract } & 1,2-Hexandiol & 2.00 & 2.00 \\
& Ethylhexylglycerin & 0.10 & 0.10 \\
\hline & Fragrance & Q.S & Q.S
\end{tabular}

\subsection{General Conditions during Measurement}

To minimize variability in skin measurements caused by exogenous factors that might affect the volunteers' general skin condition and lead to avoidable bias, severable procedures were introduced. Volunteers were asked not to smoke or to drink alcohol for $24 \mathrm{~h}$ before the measurements. In the morning, before the visits, they were not allowed to clean their face with anything but pure water or to use any cosmetic products in the area. The measurements took place in a humidity- and temperature-controlled room; the temperature of this room was $22 \pm 2{ }^{\circ} \mathrm{C}$ and the ambient humidity was $45 \pm 5 \%$. The volunteers' skin was given $2 \mathrm{~h}$ to adjust to the room conditions before measurements.

\subsection{Skin Replicas and Image Analysis}

Replicas were taken of the crow's feet area to quantify the fine variations in skin wrinkles. An adhesive paper ring (diameter, $11 \mathrm{~mm}$ ) was attached to the objective site of the crow's feet area. Care was taken to preserve the orientation of the replica with respect to the lateral canthus of the right eye to allow for accurate interpretation. Translucent silicon was mixed, in a small plastic cup, with two components: a basic substance and a catalyst (Courage \& Khazaka, Cologne, Germany). Both components contained the same concentration of blue pigment. The silicone mixture was then stirred in a vacuum pump for 15-20 s to prevent bubble formation. A layer of silicone mixture was spread over the restricted area of the adhesive paper and left to dry for $5 \mathrm{~min}$. After the silicone mixture had dried sufficiently, the specimen was labelled by subject number, initials and date and stored in a tracing paper envelope until analysis [storage conditions: $22 \pm 2{ }^{\circ} \mathrm{C}$ (temperature), $45 \pm 5 \%$ (humidity). The skin replicas were analysed using the SKIN VISIOMETER ${ }^{\circledR}$ SV 600 (Courage-Khazaka Electronic GmbH, Cologne, Germany) [8,9]. The analysis of the data yielded several parameters proportional to the degree of wrinkling, roughness and other surface markings, including R1, R2, R3, R4 and R5 (R1: skin roughness; R2: maximum roughness; R3: average roughness; R4: smoothness depth, and R5: Arithmetic Average Roughness). To ensure consistency of computer settings, lighting and other technical variables, the same operator performed image analysis of all replicas at the conclusion of the study. 


\subsection{Human Skin Primary Irritation Test}

Thirty healthy Korean subjects with Fitzpatrick skin type I, II or III were selected on the basis of inclusion and exclusion criteria, and written consent was obtained in each case. The average age was 36.0 years (range 19-50: all females). The subjects had no history of allergic contact dermatitis, nor had they used topical or systemic irritant preparations in the previous 1 month. PGLE $(0.5 \%)$ formulated with squalene was prepared and applied. The patches (chambers) stayed in place for $48 \mathrm{~h}$. Once the patches were removed, a reading was done after 48 and $72 \mathrm{~h}$ later, and the reading was scored according to modified criteria proposed by Frosch and Kligman [10] and the Cosmetic, Toiletry, and Fragrance Association (CTFA) guidelines [11] as follows: $0=$ no reaction; $1=$ slight erythema, spotty of diffuse; 2 = moderate uniform erythema; 3 = intense erythema with ethema; 4 = intense erythema with edema and vesicles. This study was approved by the Ethics Committee of the DERMAPRO/Skin Research Center, and the subjects gave written informed consent.

\subsection{Statistical Analysis}

All data are expressed as mean \pm SD. Differences between the control and treatment group were evaluated by Student's $t$ test using Statview software (Abacus Concepts, Piscataway, NJ, USA). A $p<0.01$ was considered statistically significant [12].

\section{Results}

\subsection{Ginsenosides Analysis of Panax ginseng Leaf Extract}

The high-performance liquid chromatography (HPLC) analysis revealed that PGLE contained $0.68 \%, 12.04 \%, 55.72 \%, 0.84 \%, 0.10 \%, 0.26 \%$ and $0.038 \%(\mathrm{w} / \mathrm{w})$ of ginsenoside $\mathrm{Rb} 1, \mathrm{Rb} 2, \mathrm{Rb} 3, \mathrm{Rc}, \mathrm{Rd}$, $\mathrm{Rf}$ and $\mathrm{Rh} 2$, respectively. The total amount of the seven ginsenosides in the extract was approximately $69.68 \%(w / w)$. Ginsenoside Rb2 and Rb3 were mainly contained in PGLE, at higher contents than other ginsenosides (Figure 1 and Table 2).

(A)

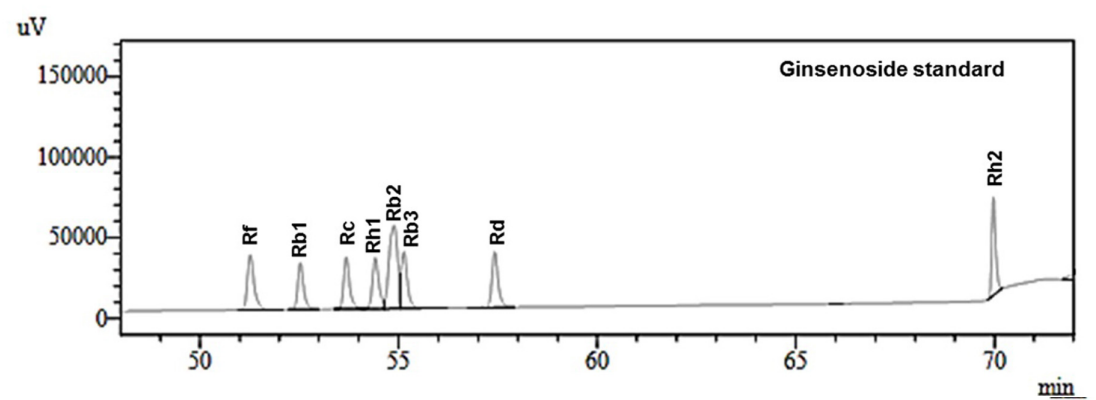

(B)

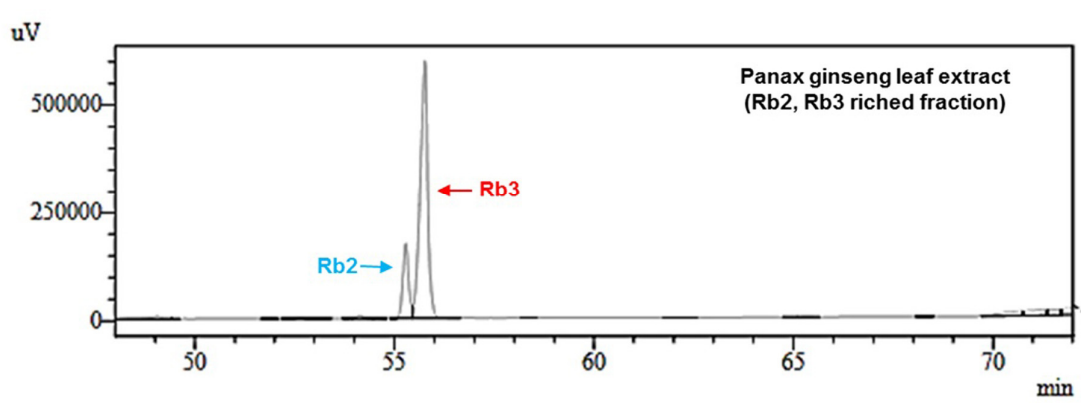

Figure 1. Phytochemical analysis of Panax ginseng leaf extract. (A) Typical HPLC chromatogram of 12 ginsenoside standards analyzed at $203 \mathrm{~nm}$; (B) Quantitative analysis of ginsenosides in Panax ginseng leaf extract. 
Table 2. Composition of Panax ginseng leaf extract (PGLE) used in this study.

\begin{tabular}{cc}
\hline Type of Ginsenosides & Content (\%) \\
\hline $\mathrm{Rb} 1$ & 0.68 \\
$\mathrm{Rb} 2$ & 12.04 \\
$\mathrm{Rb} 3$ & 55.72 \\
$\mathrm{Rc}$ & 0.84 \\
$\mathrm{Rd}$ & 0.10 \\
$\mathrm{Rf}$ & 0.26 \\
$\mathrm{Rh} 2$ & 0.038 \\
Total content of ginsenosides & 69.68 \\
\hline
\end{tabular}

\subsection{Effect of PGLE and its Two Main Ginsenosides, Rb2 and Rb3, on Type I Collagen Synthesis}

We studied the effect of PGLE on the production of human collagen type I synthesis. Figure 2A,B shows that PGLE significantly increased the production of type I collagen in human dermal fibroblast without cytotoxic effect. At a concentration of $100 \mu \mathrm{g} / \mathrm{mL}$, the extract showed $55.1 \%$ increase in collagen synthesis compared to the control.

(A)

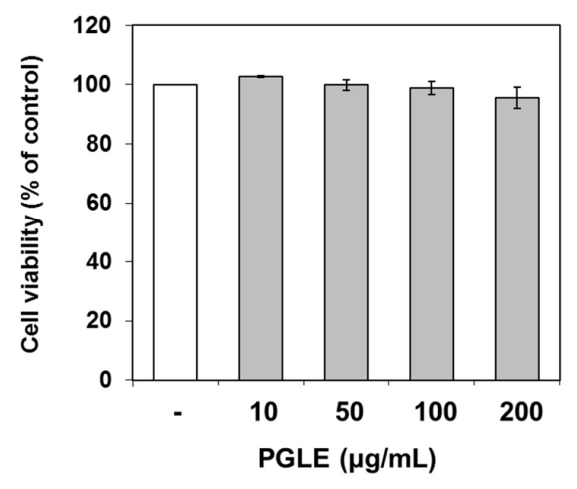

(B)

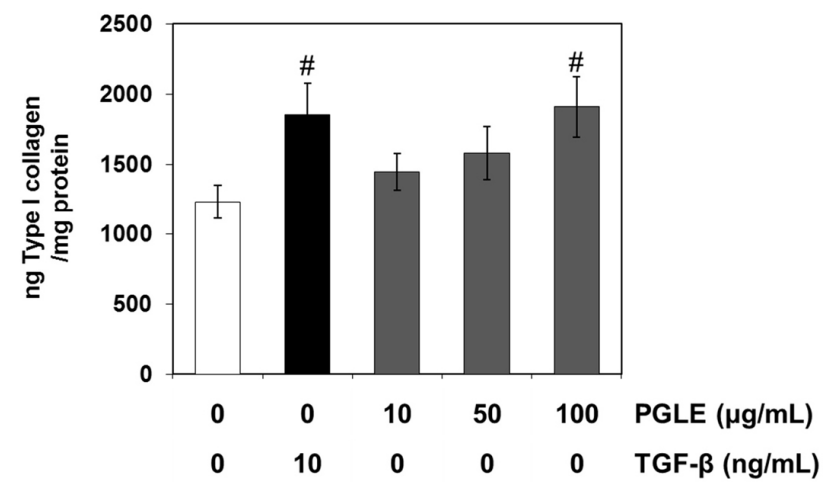

Figure 2. Effects of PGLE on collagen synthesis in human dermal fibroblast. Human dermal fibroblasts were cultured in 24-well plates. Human dermal fibroblasts were treated with or without transforming growth factor- $\beta$ (TGF- $\beta$ ) and/or PGLE at the indicated concentrations for $48 \mathrm{~h}$ at $37{ }^{\circ} \mathrm{C}$. (A) Cell viability was measured by MTT assay; (B) The culture supernatants were harvested and measured with a sandwich immunoassay kit. Data are mean \pm standard deviation. $\# p<0.01$ compared with the vehicle-treated group $(n=3)$. The results were confirmed by five independent experiments.

We further evaluated the effect of the major chemical components in PGLE on the production of human collagen type I synthesis. The results showed that Rb3 $(100 \mu \mathrm{M})$ significantly increased Type I collagen production (by $160.2 \%$ ), compared with non-treated control. However, Rb2 slightly promoted collagen synthesis in human dermal fibroblasts (Figure 3). Therefore, ginsenoside $\mathrm{Rb} 3$ may contribute to the effect of the PGLE in promoting collagen synthesis in human dermal fibroblasts. 


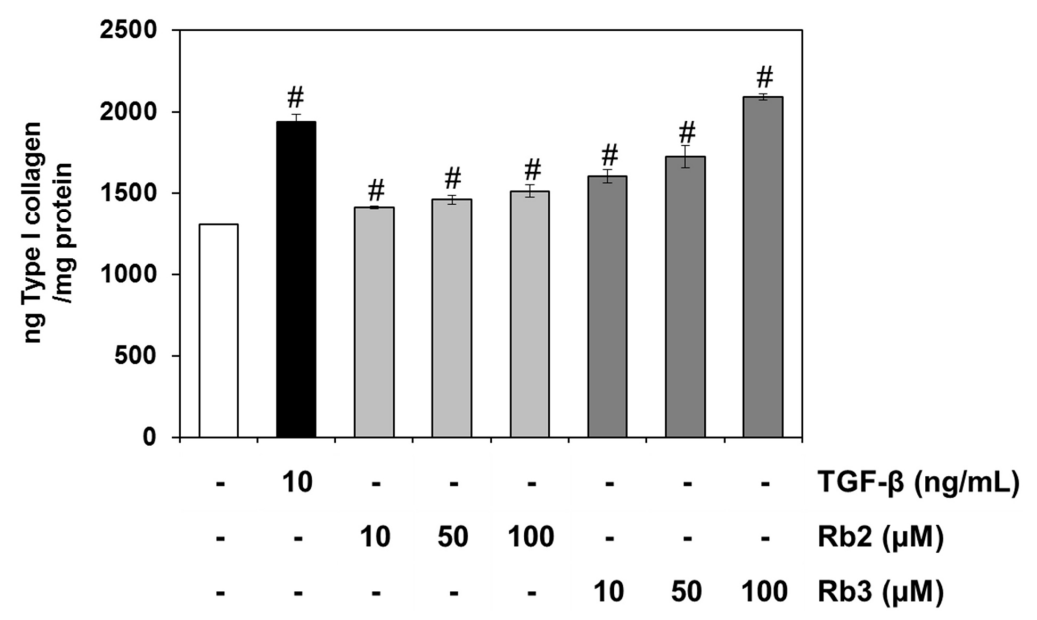

Figure 3. Effects of two main ginsenosides, $\mathrm{Rb} 2$ and $\mathrm{Rb} 3$, on collagen synthesis in human dermal fibroblasts. Human dermal fibroblasts were cultured in 24-well plates. Human dermal fibroblasts were treated with or without TGF- $\beta$ and/or ginsenosides ( $\mathrm{Rb} 2, \mathrm{Rb} 3)$ at the indicated concentrations for $48 \mathrm{~h}$ at $37^{\circ} \mathrm{C}$. The culture supernatants were harvested and measured with a sandwich immunoassay kit. Data are mean \pm standard deviation. \# $p<0.01$ compared with the vehicle-treated group $(n=3)$. The results were confirmed by five independent experiments.

\subsection{Effect of PGLE and its Two Main Ginsenosides, Rb2 and Rb3, on TGF- $\beta$ Pathway}

As TGF- $\beta$ signaling pathways perform a significant role in regulating collagen synthesis, we examined whether the TGF- $\beta$ signaling pathway can be activated by PGLE and its main ginsenosides treatments. Figure 4 shows that PGLE and Rb3 significantly increased the production of TGF- $\beta$ in human dermal fibroblast, non-treated control. However, Rb2 did not significantly promote TGF- $\beta$ production. Therefore, ginsenoside Rb3 may contribute to the effect of the PGLE in promoting TGF- $\beta$ in human dermal fibroblasts. This result suggests the possibility that Rb3 may be involved in the production of human collagen type I through TGF- $\beta$ signaling pathway.

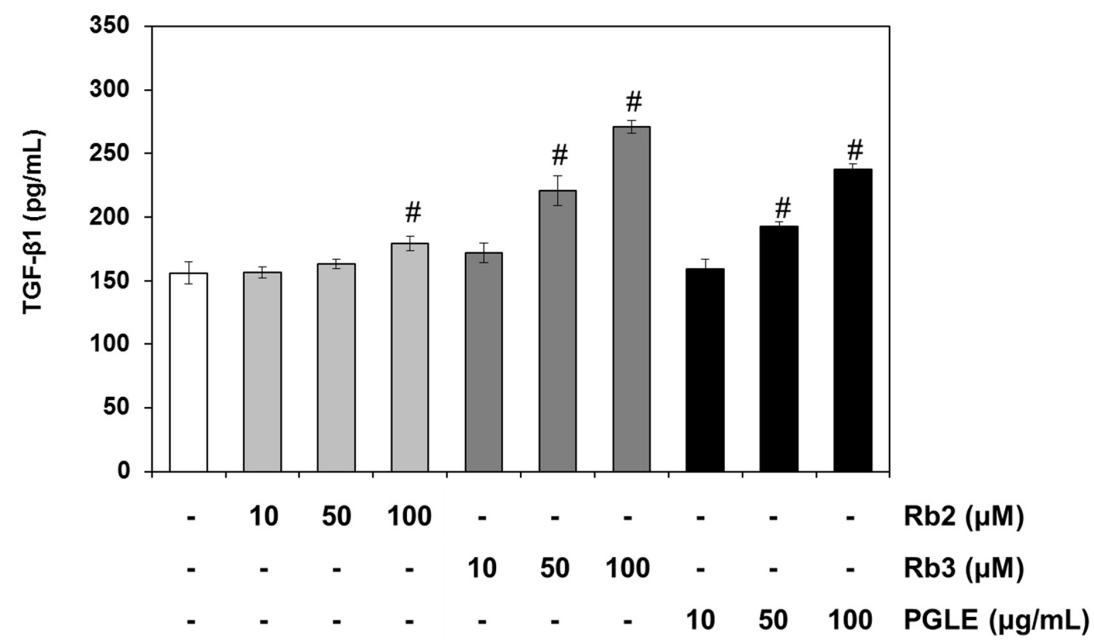

Figure 4. Effects of PGLE and its two main ginsenosides, $\mathrm{Rb} 2$ and $\mathrm{Rb} 3$, on TGF- $\beta$ production in human dermal fibroblast. Human dermal fibroblasts were cultured in 24-well plates. Human dermal fibroblasts were treated with or without PGLE and/or ginsenosides ( $\mathrm{Rb} 2, \mathrm{Rb} 3)$ at the indicated concentrations for $48 \mathrm{~h}$ at $37^{\circ} \mathrm{C}$. The culture supernatants were harvested and measured with a sandwich immunoassay kit. Data are mean \pm standard deviation. $\# p<0.01$ compared with the vehicle-treated group $(n=3)$. The results were confirmed by five independent experiments. 


\subsection{Human Skin Primary Irritation Test of PGLE}

To evaluate the irritation effect of PGLE for clinical applications to human skin, a patch test was performed. Thirty healthy Korean subjects with Fitzpatrick skin type I, II and III were selected on the basis of inclusion and exclusion criteria, and written consent was obtained in each case. The average age was 36.0 years (range 19-50: all females. Table 3 shows that in our study, none of the 30 subjects experienced a reaction based on the 48 and $72 \mathrm{~h}$ readings. Specifically, we did not observe any adverse reactions such as erythema, burning or pruritus in the study subjects that was related to the topical treatment of PGLE.

Table 3. Human skin primary irritation test.

\begin{tabular}{|c|c|c|c|c|c|c|c|c|c|c|c|c|c|c|}
\hline \multirow{2}{*}{ No } & \multirow{2}{*}{$\begin{array}{c}\text { Test } \\
\text { Material }\end{array}$} & \multicolumn{5}{|c|}{$48 \mathrm{~h}$} & \multicolumn{5}{|c|}{$72 \mathrm{~h}$} & \multicolumn{3}{|c|}{ Reaction Grade $^{(b)}$} \\
\hline & & \pm & $1+$ & $2+$ & $3+$ & $4+$ & \pm & $1+$ & $2+$ & $3+$ & $4+$ & $48 \mathrm{~h}$ & $72 \mathrm{~h}$ & Mean \\
\hline 1 & Squalene & - (a) & - & - & - & - & - & - & - & - & - & 0 & 0 & 0 \\
\hline 2 & $\begin{array}{l}\text { PGLE } \\
(0.5 \%)\end{array}$ & - & - & - & - & - & - & - & - & - & - & 0 & 0 & 0 \\
\hline
\end{tabular}

(a) No reaction; ${ }^{(\mathbf{b})}$ Reaction grade $=\sum[\{$ Grade $\times$ No. of Responders $\} /\{4$ (Maximum grade $) \times 30($ Total Subjects $\left.)\}\right] \times$ $100 \times(1 / 2)$.

\subsection{Clinical Trial on Healthy Volunteers}

In addition to the in vitro activities, the efficacy of the extract has also been clinically validated as an anti-wrinkle agent. The formulation containing PGLE at $0.05 \%$ was applied on the periorbital region of 17 volunteers over eight weeks. Our analysis of the crow's feet wrinkle showed that, compared to before treatment, there was a decrease in the depths of deep furrows at RI treated with PGLE lotion over an eight-week period $(p<0.05)$ (Figures 5 and 6). Skin wrinkle on the test site (crow's foot area of the subjects) was evaluated using SKIN VISIOMETER SV $600^{\circledR}$ (Koln, Germany). The representative data for each group are presented as R1, R2, R3, R4 and R5. Figure 6 shows that compared to before treatment, the means of all the parameters except R5 of the PGLE-treated group were significantly improved at eight weeks. These finding indicate that PGLE exerts an anti-wrinkle effect.

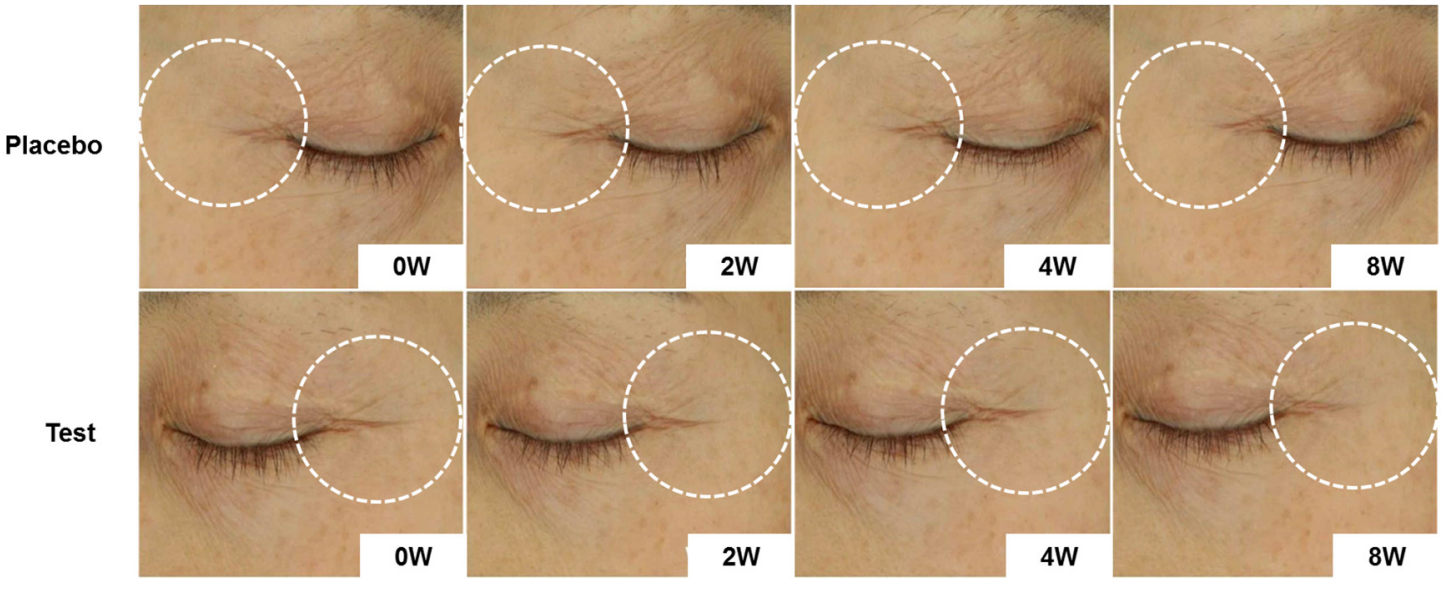

Figure 5. Visual improvement of wrinkles in the crow's feet area with PGLE application for eight weeks. Skin wrinkle images in crow's feet following eight consecutive weeks' application of the test product (Subject No. 15). 
(A)

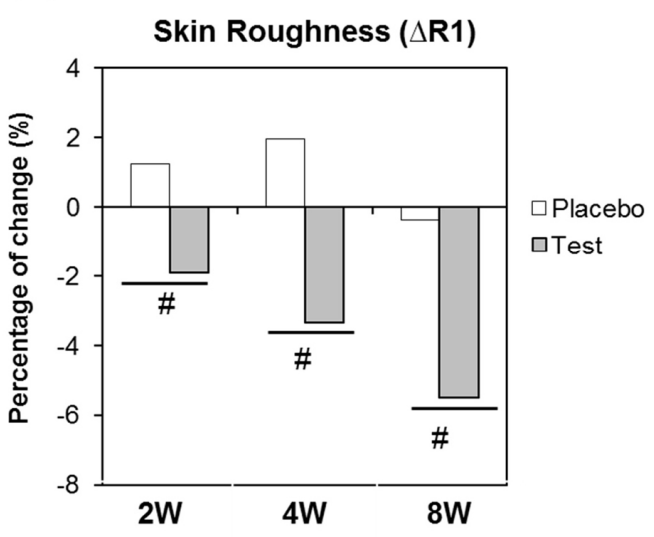

(C)

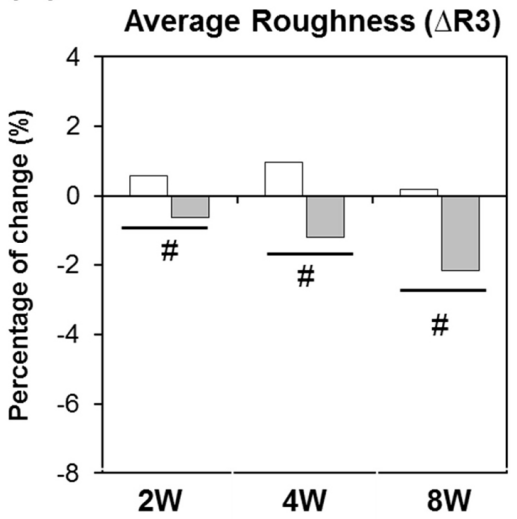

(B)

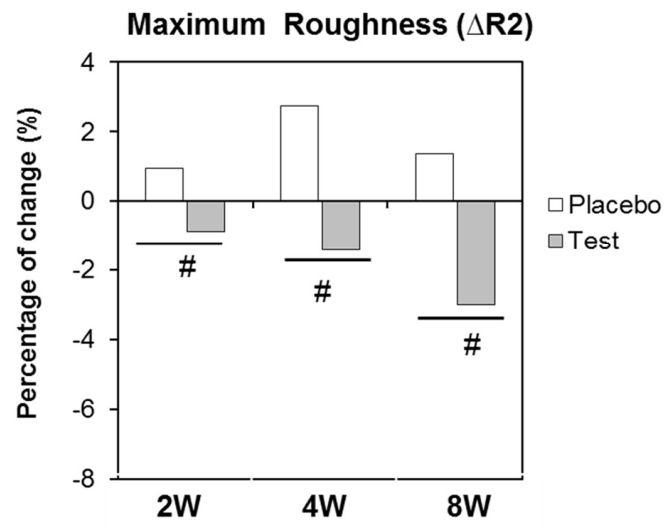

(D)

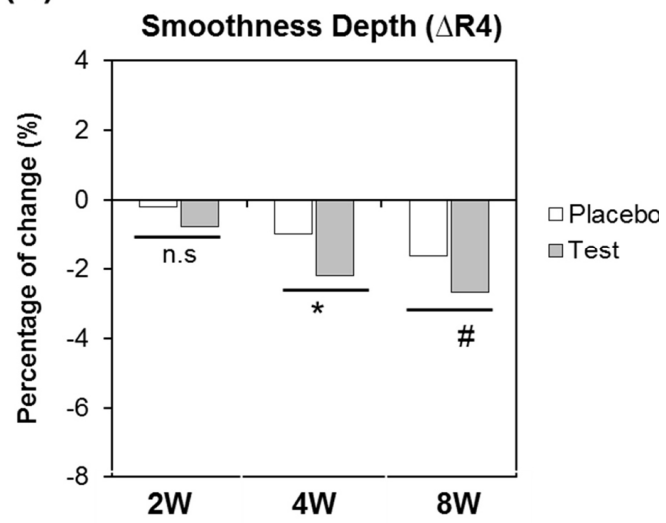

(E)

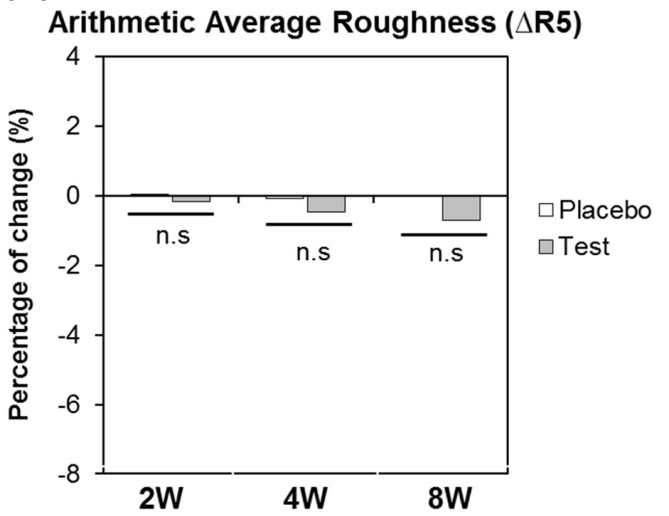

Figure 6. Changes of skin wrinkle parameters in crow's feet following eight consecutive weeks' application of test product (Mean \pm SEM. ${ }^{*} p<0.05, \# p<0.01$ vs. before treatment).

\section{Discussion}

Panax ginseng has been reported to exhibit pharmacological activities and has been used as a functional ingredient in traditional remedies to increase vitality and longevity [13]. Recent studies have focused on the development of new health products derived from ginseng; [14] most recently, ginseng roots are being considered as a valuable ingredient for skin care products [15]. A dermatological formulation containing crude extracts of ginseng has been reported to have several effects on the human and animal dermis [16-18]. Unfortunately, the ginseng root itself is too expensive to be used in external application, such as skin care products. 
Ginseng leaf has garnered less interest among scientists, although the leaves also contain a considerable amount of the active ingredients of ginseng [19,20]. Ginseng leaf has a higher content of certain ginsenosides ( $\mathrm{Rb} 2, \mathrm{Rb} 3, \mathrm{Rd}, \mathrm{Re}, \mathrm{Rh} 1$, etc.) than the roots [4,21]. However, the use of the leaf has been limited. Ginseng leaf is generally harvested every year and is often discarded without utilization. It is desirable to develop a way of utilizing the discarded ginseng leaves. In this study, we prepared purified extract (PGLE) from the dried leaves of Panax ginseng. This contained a high concentration of $\mathrm{Rb} 2$ (not less than $12 \%$ ), and $\mathrm{Rb} 3$ (not less than $55 \%$ ) mixtures.

There are several studies suggesting that the structural characteristics of collagen in the dermis are closely related to skin aging. The destruction of collagen in the dermis is responsible for a wrinkled appearance [21]. TGF- $\beta$ is the master regulator of the ECM. It is known to stimulate the fibroblasts in the dermis to synthesize several proteins such as collagen, the main constituent of dermal ECM [22]. Among the most prominent are collagen type I (80-85\%) and type III (10-15\%), which provide strength and resilience to the skin [23]. Moreover TGF- $\beta$ triggers synthesis of some components of the dermal-epidermal junction such as laminin V [24], collagen IV [25] and collagen VII [26]. Well-balanced TGF- $\beta$ signaling is therefore essential to sustain the skin in a healthy and functional condition and overall to support maintenance of a young appearance. However, the aging process affects TGF- $\beta$ levels and activity. TGF- $\beta$ has been shown to be lower both in in vitro aged fibroblasts, and in vivo aged skin [22,27], as well as in chronological skin aging and even more so in photo-aging. Thus, there is a need to restore the missing activity in aged skin conditions. The successful direct use of topical TGF- $\beta$ to treat damaged skin has been reported [28,29]. In these cases, it showed multiple benefits such as epidermal thickening, higher fibroblast density and neocollagenesis with improved skin appearance even in patients with severe photodamage.

We determined the anti-skin-aging activity of PGLE using both in vitro and in vivo models. In vitro anti-skin-aging and extract activity towards type I collagen production through the production of TGF- $\beta$ was assessed in human skin fibroblast cell cultures. We further evaluated the effect of the major chemical components in PGLE on the production of human collagen type I and TGF- $\beta$. The results showed that Rb3 significantly increased Type I collagen production and TGF- $\beta$, compared with non-treated control. However, Rb2 slightly promoted collagen synthesis and TGF- $\beta$ in human dermal fibroblast. These results suggest the possibility that $\mathrm{Rb} 3$ may be involved in the production of human collagen type I through TGF- $\beta$ signaling pathway. In addition, the possibility of PGLE as an anti-skin-aging agent has also been clinically validated. Our analysis of the crow's feet wrinkle showed that there was a decrease in the depth of deep furrows in RI treated with PGLE lotion over an eight-week period. Additional investigations are needed to further confirm the observed results as well as to elucidate the molecular mechanisms at the base of the biological activity.

Our findings demonstrate that PGLE has the potential to induce the synthesis of Type I collagen, and shows no skin primary irritation in humans. Additionally. PGLE shows good in vivo anti-skin-aging properties, probably due to the ginsenoside Rb3 content of PGLE. We suggest that PGLE can be used as a possible anti-skin-aging agent, as it prevents collagen destruction in the dermis, and the mechanisms underlying its action may be mediated via the TGF- $\beta$ signaling pathway.

\section{Conclusions}

Panax ginseng leaf extract having high levels of Rb3 shows cutaneous benefits as the basis for skin aging treatments, as indicated through in vitro assessments and skin testing in human subjects.

Acknowledgments: This study was supported by a grant from the Korean Ministry of Health and Welfare (HI15C0050).

Author Contributions: Eunsun Jung and Seoungwoo Shin conceived and designed the experiments; Seoungwoo Shin, Dahee Son and Jung-A Lee performed the experiments; Seoungwoo Shin, Eunsun Jung, and Deokhoon Park analyzed the data; Jung-A Lee, Eusun Jung and Deokhoon Park contributed reagent/materials/analysis tools; and Seoungwoo Shin and Eunsun Jung wrote the manuscript.

Conflicts of Interest: The authors declare no conflict of interest. 


\section{References}

1. Lee, J.; Jung, E.; Lee, J.; Huh, S.; Kim, J.; Park, M.; So, J.; Ham, Y.; Jung, K.; Hyun, C.G.; et al. Panax ginseng induces human Type I collagen synthesis through activation of Smad signaling. J. Ethnopharmacol. 2007, 109, 29-34. [CrossRef] [PubMed]

2. Attele, A.S.; Wu, J.A.; Yuan, C.S. Ginseng pharmacology: Multiple constituents and multiple actions. Biochem. Pharmacol. 1999, 58, 1685-1693. [CrossRef]

3. Shi, W.; Wang, Y.T.; Li, J.; Zang, H.O.; Ding, J. Investigation of ginsenosides in different parts and ages of Panax ginseng. Food Chem. 2007, 102, 664-668. [CrossRef]

4. Li, X.; Yan, Y.Z.; Kim, Y.K.; Uddin, M.R.; Bae, H.; Kim, H.H.; Park, S.U. Ginsenoside content in the leaves and roots of Panax ginseng at different ages. Life Sci. J. 2012, 9, 679-683.

5. Zhang, Y.C.; Li, G.; Jiang, C.; Yang, B.; Yang, H.J.; Xu, H.Y.; Huang, L.Q. Tissue-specific distribution of ginsenosides in different aged ginseng and antioxidant activity of ginseng leaf. Molecules 2014, 19, 17381-17399. [CrossRef] [PubMed]

6. Kim, Y.J.; Jeon, J.N.; Jang, M.G.; Oh, J.Y.; Kwon, W.S.; Jung, S.K.; Yang, D.C. Ginsenoside profiles and related gene expression during foliation in Panax ginseng Meyer. J. Ginseng Res. 2014, 38, 66-72. [CrossRef] [PubMed]

7. Li, N.; Ragheb, K.; Lawler, G.; Sturgis, J.; Rajwa, B.; Melendez, J.A.; Robinson, J.P. Mitochondrial complex I inhibitor rotenone induces apoptosis through enhancing mitochondrial reactive oxygen species production. J. Biol. Chem. 2003, 278, 8516-8525. [CrossRef] [PubMed]

8. De Paepe, K.; Lagarde, J.M.; Gall, Y.; Roseeuw, D.; Rogiers, V. Microrelief of the skin using a light transmission method. Arch. Dermatol. Res. 2000, 292, 500-510. [CrossRef] [PubMed]

9. Grove, G.L.; Grove, M.J.; Leyden, J.J.; Lufrano, L.; Schwab, B.; Perry, B.H.; Thorne, E.G. Skin replica analysis of photodamaged skin after therapy with tretinoin emollient cream. J. Am. Acad. Dermatol. 1991, 25, $231-237$. [CrossRef]

10. Frosch, P.J.; Kligman, A.M. The soap chamber test. A new method for assessing the irritancy of soaps. J. Am. Acad. Dermatol. 1979, 1, 35-41. [CrossRef]

11. Anita, S.C.; Stephen, D.G.; G N McEwen, J. CTFA Safety Testing Guideline; Cosmetic Toiletry and Fragrance Association: Washington, DC, USA, 1981; p. 20005.

12. Statview 5. A statistics Application Released for Apple Macintosh Computers; Abacus Concepts: Piscataway, NJ, USA, 2009.

13. Kim, Y.G.; Sumiyoshi, M.; Sakanaka, M.; Kimura, Y. Effects of ginseng saponins isolated from red ginseng on ultraviolet B-induced skin aging in hairless mice. Eur. J. Pharmacol. 2009, 602, 148-156. [CrossRef] [PubMed]

14. Cho, K.; Woo, H.J.; Lee, I.S.; Lee, J.W.; Cho, Y.C.; Lee, I.N.; Chae, H.J. Optimization of enzymatic pretreatment for the production of fermented ginseng using leaves, stem and roots of ginseng. J. Ginseng Res. 2010, 34, 68-75. [CrossRef]

15. Wu, J.; Zhong, J.J. Production of ginseng and its bioactive components in plant cell culture: Current technological and applied aspects. J. Biotechnol. 1999, 68, 89-99. [CrossRef]

16. Keum, Y.S.; Park, K.K.; Lee, J.M.; Chun, K.S.; Park, J.H.; Lee, S.K.; Kwon, H.; Surh, Y.J. Antioxidant and anti-tumor promoting activities of the methanol extract of heat-processed ginseng. Cancer Lett. 2000, 150, 41-48. [CrossRef]

17. Chang, L.K.; Whitaker, D.C. The impact of herbal medicines on dermatologic surgery. Dermatol. Surg. 2001, 27, 759-763. [PubMed]

18. Choi, S. Epidermis proliferative effect of the Panax ginseng ginsenoside Rb2. Arch. Pharm. Res. 2002, 25, 71-76. [CrossRef] [PubMed]

19. Xie, J.T.; Mehendale, S.R.; Wang, A.; Han, A.H.; Wu, J.A.; Osinski, J.; Yuan, C.S. American ginseng leaf: Ginsenoside analysis and hypoglycemic activity. Pharmacol. Res. 2004, 49, 113-117. [CrossRef] [PubMed]

20. Wang, H.C; Chen, C.R.; Chang, C.J. Carbon dioxide extraction of ginseng root hair oil and ginsenosides. Food Chem. 2001, 72, 505-509. [CrossRef]

21. Li, T.S.C.; Mazza, G.; Cottrell, A.C.; Gao, L. Ginsenosides in roots and leaves of American ginseng. J. Agric. Food Chem. 1996, 44, 717-720. [CrossRef] 
22. El-Domyati, M.; El-Ammawi, T.S.; Medhat, W.; Moawad, O.; Mahoney, M.G.; Uitto, J. Expression of transforming growth factor- $\beta$ after different non-invasive facial rejuvenation modalities. Int. J. Dermatol. 2015, 54, 396-404. [CrossRef] [PubMed]

23. Varani, J.; Dame, M.K.; Rittie, L.; Fligiel, S.E.; Kang, S.; Fisher, G.J.; Voorhees, J.J. Decreased collagen production in chronologically aged skin: Roles of age-dependent alteration in fibroblast function and defective mechanical stimulation. Am. J. Pathol. 2006, 168, 1861-1868. [CrossRef] [PubMed]

24. Amano, S.; Akutsu, N.; Ogura, Y.; Nishiyama, T. Increase of laminin 5 synthesis in human keratinocytes by acute wound fluid, inflammatory cytokines and growth factors, and lysophospholipids. Br. J. Dermatol. 2004, 151, 961-970. [CrossRef] [PubMed]

25. Neubauer, K.; Krüger, M.; Quondamatteo, F.; Knittel, T.; Saile, B.; Ramadori, G. Transforming growth factor-beta1 stimulates the synthesis of basement membrane proteins laminin, collagen type IV and entactin in rat liver sinusoidal endothelial cells. J. Hepatol. 1999, 31, 692-702. [CrossRef]

26. Vindevoghel, L.; Kon, A.; Lechleider, R.J.; Uitto, J.; Roberts, A.B.; Mauviel, A. Smad-dependent transcriptional activation of human type VII collagen gene (COL7A1) promoter by transforming growth factor-beta. J. Biol. Chem. 1998, 273, 13053-13057. [CrossRef] [PubMed]

27. Quan, T.; He, T.; Kang, S.; Voorhees, J.J.; Fisher, G.J. Ultraviolet irradiation alters transforming growth factor beta/smad pathway in human skin in vivo. J. Investig. Dermatol. 2002, 119, 499-506. [CrossRef] [PubMed]

28. Hussain, M.; Phelps, R.; Goldberg, D.J. Clinical, histologic, and ultrastructural changes after use of human growth factor and cytokine skin cream for the treatment of skin rejuvenation. J. Cosmet. Laser Ther. 2008, 10, 104-109. [CrossRef] [PubMed]

29. Ehrlich, M.; Rao, J.; Pabby, A.; Goldman, M.P. Improvement in the appearance of wrinkles with topical transforming growth factor $\beta_{1}$ and L-ascorbic acid. Dermatol. Surg. 2006, 32, 618-625. [CrossRef] [PubMed]

(C) 2017 by the authors. Licensee MDPI, Basel, Switzerland. This article is an open access article distributed under the terms and conditions of the Creative Commons Attribution (CC BY) license (http:/ / creativecommons.org/licenses/by/4.0/). 